hep-ph/9909514

\title{
Bi-maximal Neutrino Mixing Pattern Reexamined
}

\author{
Zhi-zhong Xing \\ Sektion Physik, Universität München, Theresienstrasse 37A, 80333 München, Germany \\ Electronic address: xing@theorie.physik.uni-muenchen.de
}

\begin{abstract}
I propose a simple extension of the bi-maximal neutrino mixing pattern, allowing slight coupling between solar and atmospheric neutrino oscillations as well as large $C P$ violation. The new scenario is compatible with either the vacuum oscillation solution or the large-angle MSW solution to the solar neutrino problem.
\end{abstract}

PACS number(s): 14.60.Pq, 13.10.+q, 25.30.Pt 
To interpret current experimental data on atmospheric and solar neutrino oscillations, a lot of interest has been paid to the "bi-maximal" neutrino mixing pattern (up to a trivial sign rearrangement) [1]:

$$
U=\left(\begin{array}{ccc}
\frac{1}{\sqrt{2}} & \frac{1}{\sqrt{2}} & 0 \\
-\frac{1}{2} & \frac{1}{2} & \frac{1}{\sqrt{2}} \\
\frac{1}{2} & -\frac{1}{2} & \frac{1}{\sqrt{2}}
\end{array}\right)
$$

Note that $U$ links the neutrino mass eigenstates $\left(\nu_{1}, \nu_{2}, \nu_{3}\right)$ to the neutrino flavor eigenstates $\left(\nu_{e}, \nu_{\mu}, \nu_{\tau}\right)$.

Different from the "tri-maximal" [2] or "democratic" (nearly bi-maximal) [3] neutrino mixing scenarios, the vanishing of $U_{e 3}$ in the bi-maximal mixing scenario assures the absence of $C P$ violation and an exact decoupling between solar $\left(\nu_{e} \rightarrow \nu_{\mu}\right)$ and atmospheric $\left(\nu_{\mu} \rightarrow \nu_{\tau}\right)$ neutrino oscillations with identical mixing factors (i.e., $\sin ^{2} 2 \theta_{\text {sun }}=\sin ^{2} 2 \theta_{\text {atm }}=1$ ). At present this special ansatz seems to be more favored by the vacuum oscillation solution than by the large-angle MSW solution to the solar neutrino problem.

In this short note I propose a simple extension of the naive bi-maximal neutrino mixing pattern given above, so as to accommodate possible large $C P$ violation in the lepton sector and to allow much flexibility in accounting for the solar neutrino problem. The new lepton flavor mixing matrix takes the following form 1 :

$$
V=\left(\begin{array}{ccc}
\frac{c}{\sqrt{2}} & \frac{c}{\sqrt{2}} & -i s \\
-\frac{A}{2} & \frac{A^{*}}{2} & \frac{c}{\sqrt{2}} \\
\frac{A^{*}}{2} & -\frac{A}{2} & \frac{c}{\sqrt{2}}
\end{array}\right),
$$

where $s \equiv \sin \theta, c \equiv \cos \theta$, and $A=1+i s$. The mixing angle $\theta$ measures a slight coupling between solar and atmospheric neutrino oscillations, whose mass-squared differences are

$$
\begin{aligned}
& \Delta m_{\text {sun }}^{2}=\left|m_{2}^{2}-m_{1}^{2}\right|, \\
& \Delta m_{\text {atm }}^{2}=\left|m_{3}^{2}-m_{2}^{2}\right|,
\end{aligned}
$$

respectively. The "observed" hierarchy $\Delta m_{\text {sun }}^{2} \ll \Delta m_{\text {atm }}^{2}$ represents our today's understanding of the neutrino mass spectrum 9 .

Clearly $U$ can be reproduced from $V$ with $\theta=0$. The rephasing-invariant strength of $C P$ violation turns out to be

\footnotetext{
${ }^{1}$ Here only the Dirac-type $C P$ phase is taken into account, as the Majorana-type ones have no effect in neutrino oscillations.

${ }^{2}$ Throughout this work we do not take the LSND evidence for neutrino oscillations [4], which was not confirmed by the KARMEN experiment [5], into account.
} 


$$
\begin{aligned}
\mathcal{J} & =\operatorname{Im}\left(V_{i \alpha} V_{j \beta} V_{i \beta}^{*} V_{j \alpha}^{*}\right) \\
& =\frac{s c^{2}}{4}
\end{aligned}
$$

in which $(i, j)$ run over $(e, \mu),(\mu, \tau)$ or $(\tau, e)$ and $(\alpha, \beta)$ over $(1,2),(2,3)$ or $(3,1)$. The mixing factors of solar $\left(\nu_{e} \rightarrow \nu_{e}\right.$ disappearance) and atmospheric $\left(\nu_{\mu} \rightarrow \nu_{\mu}\right.$ disappearance) neutrino oscillations read

$$
\begin{aligned}
\sin ^{2} 2 \theta_{\text {sun }} & =c^{4}, \\
\sin ^{2} 2 \theta_{\text {atm }} & =1-s^{4} .
\end{aligned}
$$

The correlation between $\mathcal{J}$ and $\left|V_{e 3}\right|^{2}$ is illustrated in Fig. 1(a) for $\left|V_{e 3}\right|^{2} \leq 0.1$, and that between $\sin ^{2} 2 \theta_{\text {sun }}$ (or $\sin ^{2} 2 \theta_{\text {atm }}$ ) and $\left|V_{e 3}\right|^{2}$ is shown in Fig. 2(b). Some comments are in order.

- The strength of $C P$ violation in this new neutrino mixing scenario can be as large as few percent. A signal of $C P$ (or $T$ ) violation could be measured from the probability asymmetry between $\nu_{\mu} \rightarrow \nu_{e}$ and $\bar{\nu}_{\mu} \rightarrow \bar{\nu}_{e}$ (or $\nu_{e} \rightarrow \nu_{\mu}$ ) in the future long-baseline neutrino experiments with $L \sim E / \Delta m_{\text {sun }}^{2}$. Such a measurment is feasible if and only if the large-angle MSW oscillation is the true solution to the solar neutrino problem.

- The mixing factor of solar neutrino oscillations is sufficiently large, compatible with either the large-angle MSW solution or the vacuum oscillation solution to the solar neutrino problem [6].

- The mixing factor of atmospheric neutrino oscillations is nearly maximal for reasonable values of $\left|V_{e 3}\right|^{2}$. This result is particularly favored by the Super-Kamiokande [7] and CHOOZ [8] experiments.

It is also worth pointing out that the lepton flavor mixing matrix $V$ is symmetric about its axis $V_{e 3^{-}}-V_{\mu 2}-V_{\tau 1}$. In comparison, the quark flavor mixing matrix (i.e., the CKM matrix) is approximately symmetric about its axis $V_{u d^{-}} V_{c s^{-}} V_{t b}$ [9]. This qualitative difference in the textures of quark and lepton mixing matrices could have a yet unknown dynamical reason.

There are of course some other possibilities, depending on the number of free parameters to be introduced into $U$, towards a slight modification of the exactly bi-maximal neutrino mixing pattern. In Ref. [10], for instance, $U$ has been modified to $U^{\prime}=R_{12}^{\mathrm{T}}\left(\theta_{12}\right) U$, where $R_{12}\left(\theta_{12}\right)$ describes a small rotation in the real $(1,2)$ plane to diagonalize the charged lepton mass matrix $\left(\theta_{12} \approx \arcsin \sqrt{m_{e} / m_{\mu}} \approx 4^{\circ}\right)$. This scenario, in which $C P$ symmetry remains conserved, predicts $\left|U_{e 3}^{\prime}\right|^{2} \approx 0.002, \sin ^{2} 2 \theta_{\text {sun }} \approx 0.985$, and $\sin ^{2} 2 \theta_{\text {atm }} \approx 1.000$. It is therefore distinguishable from the new mixing pattern $V$ proposed in Eq. (2), through the delicate detection of leptonic $C P$ violation in the long-baseline neutrino experiments or through the accurate determination of mixing factors in the atmospheric and solar neutrino experiments. In Ref. [11] the author presented a different nearly bi-maximal neutrino mixing ansatz, based on the arguments of lepton-quark similarities and the assumed textures of charged lepton and neutrino mass matrices. Such an ansatz involves several free parameters, whose values are completely unknown. Hence its interpretation of current neutrino oscillation data relies 
somehow on the fine-tuning of those parameters, and its consequences on the long-baseline neutrino oscillations are not as unique as those of the simpler and more instructive scenarios discussed above.

Finally I should emphasize that the bi-maximal mixing pattern $U$ in Eq. (1) and its revisited version $V$ in Eq. (2) require some special flavor symmetries to be imposed on the charged lepton and neutrino mass matrices [3]. It is more likely that $V$ (or $U$ ) serves as the leading-order approximation of a more complicated flavor mixing matrix. At present, however, such a simplified scenario is very instructive and useful to explore the main features of lepton flavor mixing and $C P$ violation through neutrino oscillations. 


\section{FIGURES}
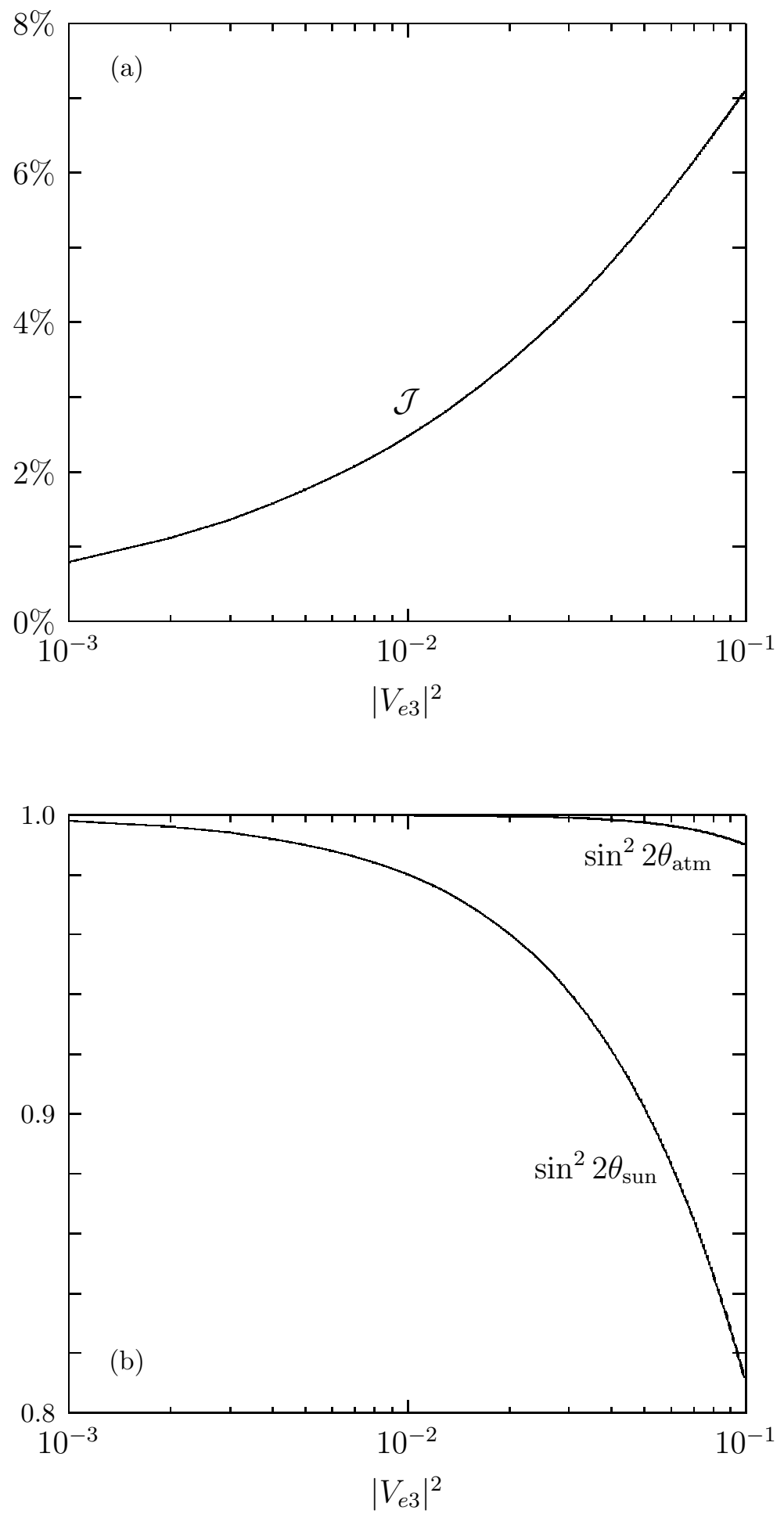

FIG. 1. Illustrative plots for the correlation between $\mathcal{J}, \sin ^{2} 2 \theta_{\text {sun }}$ or $\sin ^{2} 2 \theta_{\text {atm }}$ and $\left|V_{e 3}\right|^{2}$ in the new neutrino mixing scenario. 


\section{REFERENCES}

[1] V. Barger, S. Pakvasa, T.J. Weiler, and K. Whisnant, Phys. Lett. B 437, 107 (1998).

[2] N. Cabibbo, Phys. Lett. B 72, 333 (1978). For a recent look at this scenario, see: W.G. Scott, hep-ph/9909431.

[3] H. Fritzsch and Z.Z. Xing, Phys. Lett. B 372, 265 (1996); Phys. Lett. B 440, 313 (1998); hep-ph/9909304.

[4] C. Athanassopoulos et al., Phys. Rev. Lett. 75, 2650 (1995).

[5] B. Zeitnitz, talk given at Neutrino '98, Takayama, Japan, June 1998.

[6] J.N. Bahcall, P.I. Krastev, and A.Y. Smirnov, Phys. Rev. D 58, 096016 (1998); For a brief review with extensive references, see: S.T. Petcov, hep-ph/9907216.

[7] Y. Fukuda et al., Phys. Lett. B 436, 33 (1998); Phys. Rev. Lett. 81, 1562 (1998); Y. Suzuki, talk given at the 17th International Workshop on Weak Interactions and Neutrinos, Cape Town, South Africa, January 1999; and references therein.

[8] M. Apollonio et al., Phys. Lett. B 420, 397 (1998).

[9] Z.Z. Xing, Phys. Rev. D 51, 3958 (1995); Nucl. Phys. B (Proc. Suppl.) 50, 24 (1996).

[10] M. Jezabek and Y. Sumino, Phys. Lett. B 457, 139 (1999); and references therein.

[11] B. Stech, hep-ph/9905440, and hep-ph/9909268. 